\title{
Innovative Large Scale Wireless Sensor Network Architecture Using Satellites and High-Altitude Platforms
}

\author{
Yasser Albagory ${ }^{\mathrm{a}}$, Fahad Al Raddady ${ }^{\mathrm{b}}$, Sultan Aljahdali ${ }^{\mathrm{c}}$ and Omar Said ${ }^{\mathrm{d}}$ \\ ${ }^{a}$ Department of Information technology, College of Computers and Information technology, Taif University, \\ Saudi Arabia \\ ${ }^{b}$ Department of Computer Science, College of Computers and Information technology, Taif University, Saudi \\ Arabia \\ ${ }^{c}$ Department of Information technology, College of Computers and Information technology, Taif University, \\ Saudi Arabia \\ ${ }^{d}$ Department of Information technology, College of Computers and Information technology, Taif University, \\ Saudi Arabia
}

\begin{abstract}
Wireless sensor network has many applications and very active research area. The coverage span of this network is very important parameter where wide coverage area is a challenge. This paper proposes an architecture for large-scale wireless sensor network (LSWSN) based on satellites and the High-Altitude Platforms (HAP) where the sensor nodes are located on the ground and a wide coverage sink station may be in the form of a satellite or a network of HAPs. A scenario is described for multilayer LSWSN and a study for the system requirements has been established showing the number of Satellites, HAPs and coverage per each sink according to the elevation angle requirements. The Satellite-HAP-Sensor multilayer LSWSN architecture has the feasibility for effective energy and earth coverage and is optimum for covering largely sparse regions.
\end{abstract}

Index Terms: High-Altitude platforms, Satellite Communications, HAP, Wireless Sensor Networks.

(C) 2014 Published by MECS Publisher. Selection and/or peer review under responsibility of the Research Association of Modern Education and Computer Science

\section{Introduction}

Wireless sensor network (WSN) becomes an important area of research that has gained attention in the last years where it has many applications including industrial manufacturing, military, agriculture, health care, and security [1-4]. There are many challenges for the WSN such as the limited battery life time, wireless communication performance, routing and its data security [5-6]. The ground WSN has a very limited range due

* Corresponding author.

E-mail address: dalbagory@gmail.com; alraddady@tu.edu.sa; aljahdali@tu.edu.sa; o.saeed@tu.edu.sa 
to the communications impairments especially the fading problems where the radius of the sink coverage is limited to a few meters for most of the existing sensors technology. Recently, the feasibility of covering large area WSN [7] with High-Altitude Platforms (HAPs) has been proved for the existing sensors technologies without using sensor power enhancements or external power sources and the coverage radius may extend to several tenses of kilometers. The HAPs provide many attractive features and superior performance compared to the conventional terrestrial or satellite technology [8] such as the line-of-sight communications and reduced time delays. The HAP and satellite technologies make it possible for Large-Scale Wireless Sensor Network (LSWSN) establishment where the whole earth surface can be monitored for several various applications including monitoring of weather, crop, and climate changes.

Satellite WSN requires special sensors with parabolic antennas for efficient transmission to satellites and this indicates that a continuous and reliable power source should be available. Consequently, the sensor in satellite sensor network will be easily detected resulting in a reduced security of the system. Therefore, to provide a LSWSN, there should be a means to use the existing low power sensors and at the same time, a large coverage area should be maintained. This gap can be closed by applying the HAP technology in the WSN in conjunction with satellites producing Satellite-HAP-Sensors network.

The paper is organized as follows. In section 2, the related work considering HAPs or satellites in WSNs is summarized; in particular, some of the main shortcomings of satellite broadband communication systems are recalled. In Section 3, the different LSWSN architectures are introduced. In Section 4, a comparison between these architectures is demonstrated. Finally conclusion is introduced in Section 5.

\section{Related Works}

There are many literatures which apply satellite and HAPs in sensor networks. The work in [9] has deployed unmanned aerial vehicles (UAVs) connected wirelessly with cameras in disaster management applications. The UAV flay over the disaster area such as large traffic accident and wood fire, and get images or videos by high quality data sensor. Hence, the collected information is handled and delivered to the end user of final destination. This research presents optimal sensors positions as a challenge and formulates this problem to be an integral linear program.

There is another proposed two types of cell distribution with HAP system. The coverage area of the HAP is divided into one or multiple cells. [10-11] proposed an architecture in which the HAP coverage area has been divided into 121 and 19 cells to improve the HAP system capacity. Based on the [10] architecture of HAPs and WSN, there were two proposed configurations for using HAPs in WSN systems for different applications. In the first scenario, the information of sensor nodes is transmitted directly to the HAP. This scenario reduced the complexity and enhanced overall energy consumption in WSN, which makes it suitable for some special WSN applications such as low data transmission in large coverage area applications. In the second proposed scenario, the sensor nodes are organized into clusters inside the HAP cell. A special node, which has higher-energy, is selected as the cluster head. The information, which is collected by senor nodes, is sent to the cluster head, which is responsible to send all data to the HAP. Sensors energy was reserved and distance from the cluster head are two important factors in cluster formation. Also, this scenario has reduced the complexity and energy consumption of all sensor nodes. So, it became more suitable for multimedia WSN applications which required high data transmission channels. Also, [12-13] proposed additional work, which is related to use HAPs in order to enhance WSN efficiency.

\section{The Proposed LSWSN Architecture}

The objectives of the proposed environmental monitoring scenarios are retrieving of physical quantities such as humidity and temperature over a wide area that can be measured by scalable and reliable WSN architectures. The proposed WSN architecture consists of four layers; that is Satellite-HAP-WSN.

Sometimes, it is required to cover some large isolated areas that may be in different continents and 
establishing a communication link that may be impossible even with the previous HAP-Sensors architecture. The ideal solution in this situation is to use a satellite for closing this communication gap. This architecture consists of four layers; the first is a global linking satellite acting as a global sink, the second is HAP located at even higher altitudes may be near $50 \mathrm{~km}$ high, the third is another lower altitude HAP acting as a first sink, and the fourth is the sensors that may be on the ground or located on the lower HAP. The sink in this architecture consists of two layers. This architecture can be seen as multilayer sink LSWSN and where the satellites are used only at the sparse regions to be covered as shown in Fig. 1.

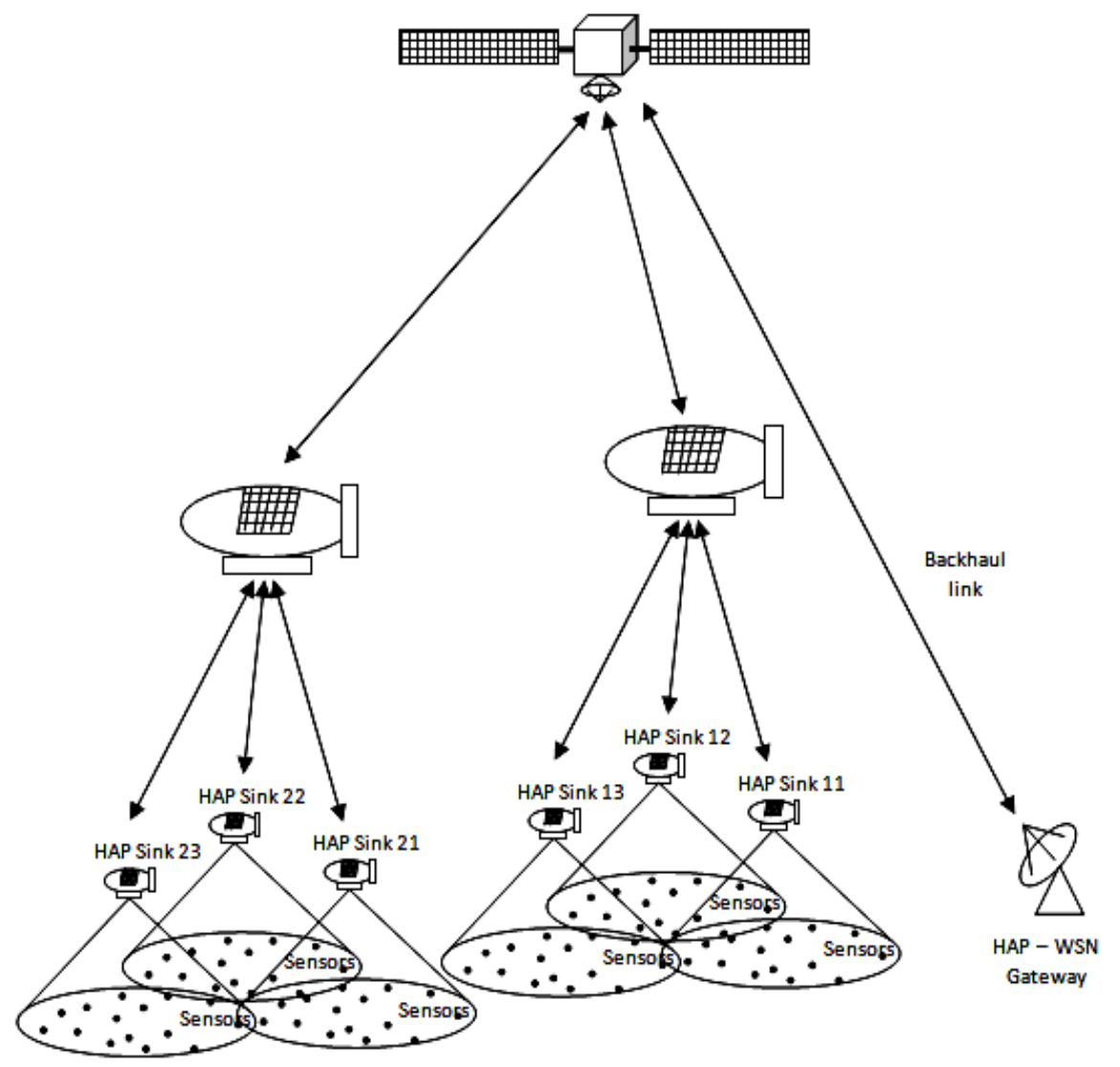

Fig. 1: Satellite-HAP-Sensor architecture

\section{Geometrical Study for LSWSN Architecture}

In this section, the scenario introduced in the last section is analyzed for global coverage. The number of required satellites and HAPs are determined in each scenario in a sense that cellular coverage is used. For a satellite or HAP station located at an altitude of $\mathrm{h} \mathrm{km}$, covering an area of a minimum elevation angle of $\mathrm{E}$, the area of this footprint as shown in Fig. 2 is given by:

$$
A=2 \pi R_{e}{ }^{2}(1-\cos (\theta))
$$

where 


$$
\theta=\left[\cos ^{-1}\left(\frac{R_{e} \cos (E)}{R_{e}+h}\right)\right]-E
$$

where $R_{e}$ is the earth radius (approximately $6378 \mathrm{~km}$ ).

For a cellular WSN coverage shown in Fig. 3, the cell is modeled as a hexagonal shape with radius $R_{c}$ which makes a circle of an area $\mathrm{A}=\pi R_{c}{ }^{2}$. The circular footprints are tessellated with overlapping areas so we can treat the actual cells distribution using the hexagonal shape rather than the circular one. For the hexagonal cell of radius $R_{c}$, the cell area $\mathrm{A}_{\mathrm{H}}$ is given by:

$$
A_{H}=\frac{3 \sqrt{3}}{2} R_{c}^{2}
$$

To cover the whole earth surface with SAT/HAP WSN, we need a number of SAT/HAP stations that is given by:

$$
N_{\text {SAT/HAP }}=\left\lceil\frac{\text { Earth Surface Area }}{\text { Station Coverage Area }}\right\rceil
$$

Where $\lceil$.$\rceil denotes to an approximation to the nearest higher integer value.$

Fig. 4 displays the total number of stations (SAT or $H A P)$ at different elevation angles required per station. The satellite altitudes are chosen to at $800 \mathrm{~km}$ high. For HAPs, the altitudes are chosen at $20 \mathrm{~km}$. The results show that the number of required Satellites is much less than that of HAPs due even for satellite due to the higher altitudes and this number becomes very large at higher elevation. The number of HAPs can be reduced by increasing its coverage area or reducing the required elevation angle.

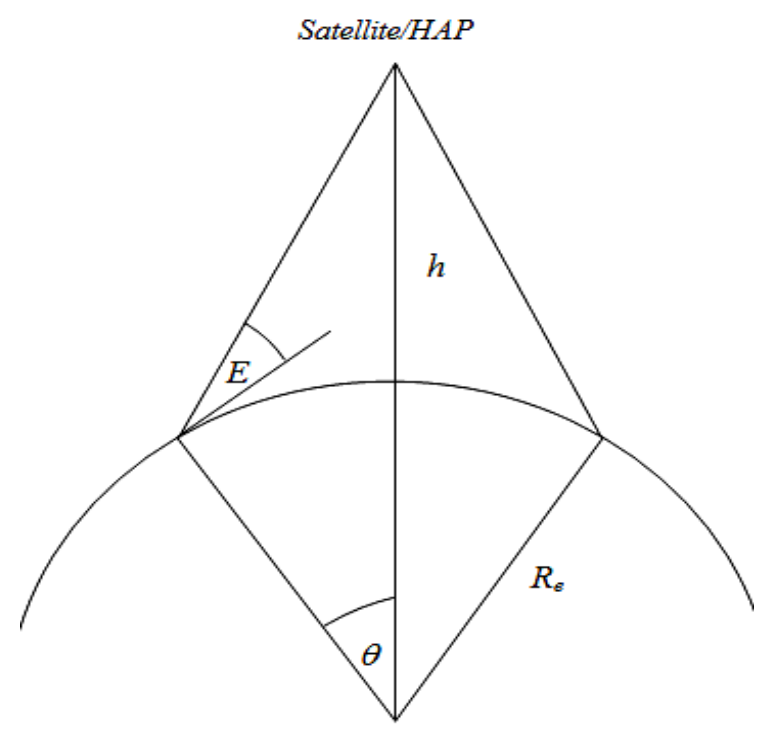

Fig. 2: Geometry of Satellite/HAP sink coverage showing the elevation angle and sink height. 


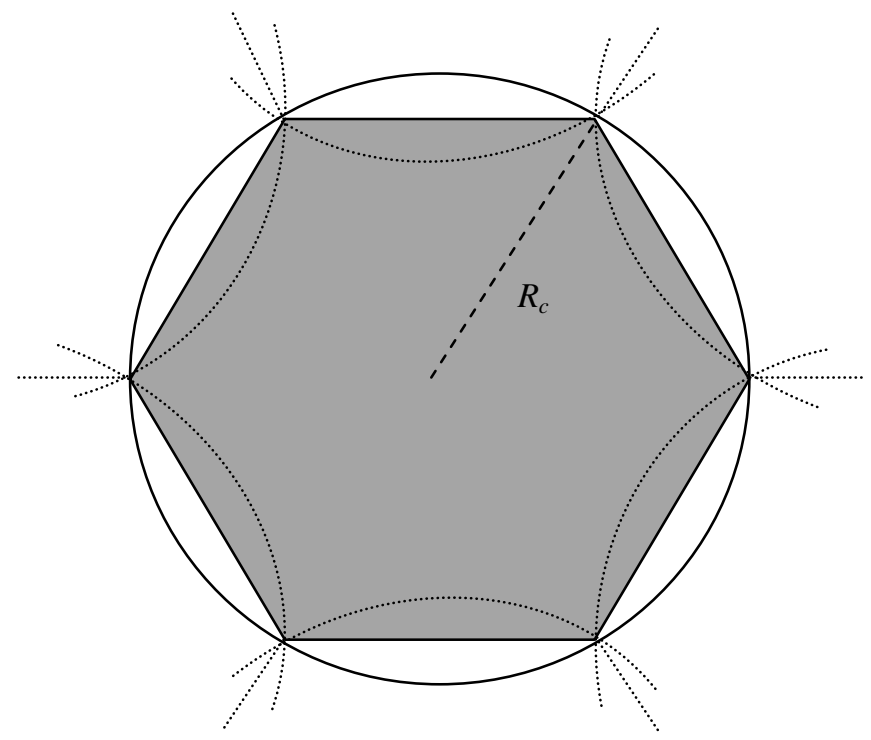

Fig. 3: Cell dimensions of Satellite/HAP sink

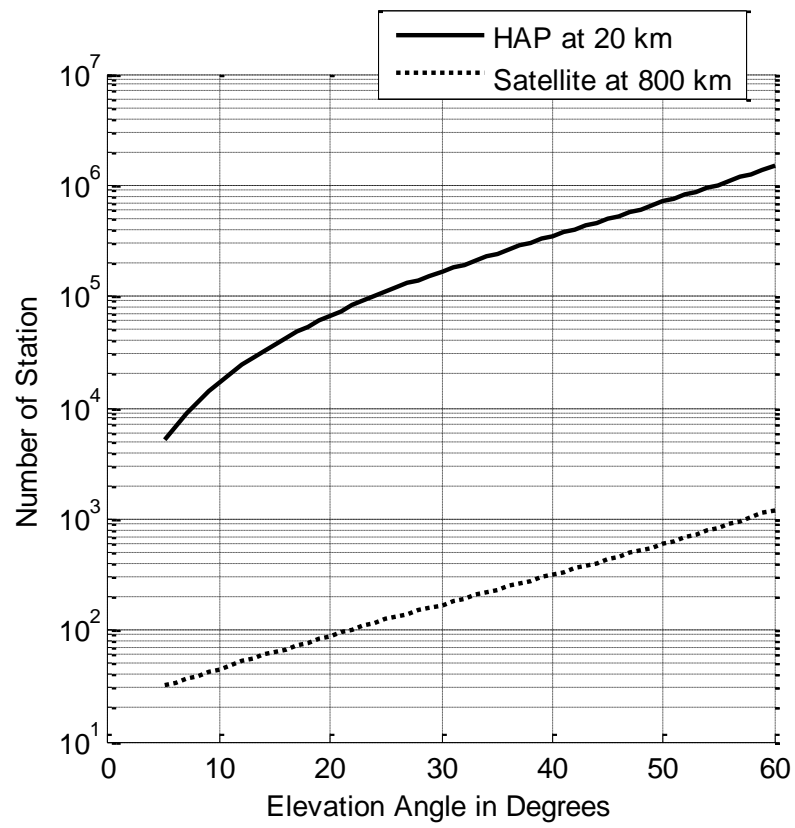

Fig. 4: The variation of the number of Satellite and HAP sink stations for LSWSN using HAPs at $20 \mathrm{~km}$ and Satellites at $800 \mathrm{~km}$ high with the acceptable elevation angle. 


\section{Conclusion}

This paper has presented an architecture for Large-Scale wireless sensor network (LSWSN) where the whole earth surface can be covered using satellites and high-altitude platforms (HAPs). The architecture comprises of global satellite main sink station with lower layer of HAP sink stations. The architecture has been defined and each layer in the system has been described. This LSWSN architecture have been proved to cover the whole earth surface, it can be established by using either satellites and/or HAPs where for HAPs only based WSN we need a great number of HAPs while using satellites will reduce this number and the network becomes feasible especially when we need to cover specific sparse regions that are difficult to link by HAPs.

\section{References}

[1] Yun Wang, Matthew Wilkerson, Xudong Yu, Hybrid Sensor Deployment for Surveillance and Target Detection in Wireless Sensor Networks, International Conference on Wireless Communications and Mobile Computing (IWCMC), Istanbul, Turky, Pages: 326 - 330, 2011.

[2] Alageswaran, R., Usha, R.; Gayathridevi, R.; Kiruthika, G., Design and implementation of dynamic sink node placement using Particle Swarm Optimization for life time maximization of WSN applications, IEEE International Conference on Advances in Engineering, Science and Management (ICAESM), Nagapattin, India, Pages: 552 - 555, 30-31 March 2012.

[3] Salminen, J., Andrey, L., Yla-Jaaski A., Smart Trigger for Ultralow Power and Time Critical WSN Applications, IEEE International Conference on Green Computing and Communications (GreenCom), Besançon, France, 20-23 Nov. 2012.

[4] Weijun Yu , Xianyi Qian Design of 3KW Wind and Solar Hybrid Independent Power Supply System for 3G Base Station. International Symposium on Knowledge Acquisition and Modeling, Wuhan, China, 2009, Pages: 289 - 292.

[5] Fei Yin, Zhenhong Li, Haifeng Wang, Energy-efficient data collection in multiple mobile gateways WSNMCN convergence system, IEEE International Conference on Consumer Communications and Networking Conference (CCNC), Las Vegas, AA, USA, Pages: 271 - 276, 2013.

[6] Hui Suoa, Jiafu Wan, Caifeng Zoua, Jianqi Liu, "Security in the Internet of Things: A Review", International Conference on Computer Science and Electronics Engineering, Pages: 648-651, Guangzhou, China, 2012.

[7] Urso, M.F., Mondin, M.; Falletti, E.; Sellone, F.; Arnon, S., Self Organizing WSN Collaborative Beamforming for HAP Communications, IEEE GLOBECOM Workshops, LA, USA, Pages: 1 - 5, Nov. 30 -Dec. 42008.

[8] N. Celandroni1, et al., 2012, A Survey Of Architectures And Scenarios In Satellite-Based Wireless Sensor Networks: System Design Aspects, International Journal Of Satellite Communications And Networking, Volume 31 Issue 1, Pages: 1-38.

[9] M. Quaritsch, K. Kruggl, D. Strucl, S. Bhattacharya, M. Shah, B. Rinner, 2010, Networked UAVs as aerial sensor network for disaster management applications, Elektrotechnik und Informationstechnik, Volume 127, Issue 3, Pages: 56-63.

[10] Z. Yang, A. Mohammed, High Altitude Platforms for Wireless Sensor Network applications, IEEE international conference on Wireless Communication Systems (ISWCS '08), Reykjavik, Iceland, pages: 613-617, 21-24 Oct. 2008.

[11] Z. Yang, A. Mohammed, A Study of Multiple Access Schemes for Wireless Sensor Network Applications via High Altitude Systems, IEEE International Conference on Vehicular Technology Conference, Barcelona, Spain, Pages: 1 - 5, 26-29 April 2009. 
[12] Paul Daniel Mitchell, Jian Qiu, Hengguang Li, David Grace, 2010, Use of aerial platforms for energy efficient medium access control in wireless sensor networks, Computer Communication, Volume 33, Issue 4, Pages 500 - 512.

[13] K. Daniel, S. Rohde, N. Goddemeier, C. Wietfeld, Channel Aware mobility for self organizing wireless sensor swarms based on low altitude platforms, IEEE International Symposium on Wireless Communication Systems (ISWCS), United Kingdom, Pages: 145 - 149, 19-22 Sept. 2010.

\section{Authors Profile}

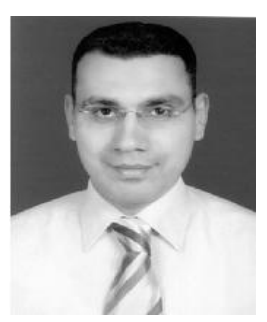

Yasser Albagory: Born in 1975, B.Sc in Electronic Engineering in 1998 and the M.Sc in adaptive arrays for mobile radio communications in 2002 from the Faculty of Electronic Eng., Egypt. He also has been awarded the Ph.D degree in Communications Engineering in the field of High-Altitude Platform Wireless Communications System in 2008. Now, he is an assistant professor at the Information Technology Department, College of Computers and Information Technology, Taif University, Saudi Arabia. The research interests include adaptive antenna arrays, mobile communications, and high altitude platforms, satellite communications, and digital communications. He is a reviewer of many international conferences and journals in the field of wireless communications and has many journal papers in the area of smart antennas and high-altitude platforms. He is one of the editorial board of the Asian Journal of Technology \& Management Research and International Journal of Technology \& Management Research. He judges many technical issues regarding the installation of mobile base stations and their effects on the surrounding environment in Egypt. In addition, he is an author of two books in the field of high-altitude platforms and their role in cellular communications issued in 2013.

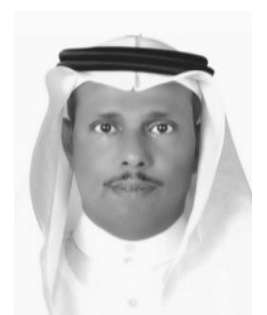

Fahad Alraddady: Master of Science in personal mobile and satellite communications in 2002 and Doctor of Philosophy in Telecommunication Network from the University of Bradford, Bradford, U.K, 2008. He also has been certified in Quality Management Systems Requirement (ISO 9001), PME Jeddah, Saudi Arabia. His research interests include mobile communications, satellite communications, high-altitude platforms communications, and digital signal processing. He has many papers in the field of queue management algorithms for Internet congestion control. Dr. Fahad was the vice dean of quality affairs in the college of Computers and Information Technology, Taif University, Saudi Arabia. Now, he is the dean of the College of Computers and Information Technology, Taif University, Saudi Arabia.

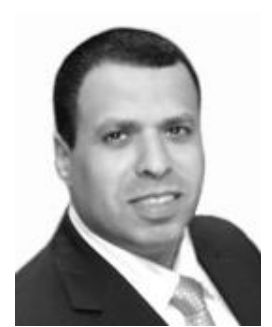

Sultan Aljahdali is the Dean of the College of Computers and Information Technology at Taif University, Kingdom of Saudi Arabia. Before joining Taif University, he has served as Information Technology Manager at General Authority of Civil Aviation and Saudi Airlines respectively. Dr. Aljahdali received the B.S from Winona State University, M.S. with honor from Minnesota State University Mankato, and Ph.D., in Information Technology from George Mason University in Fairfax, Virginia. He is the recipient of the prestigious higher education scholarship from the government of Saudi Arabia for pursuing his B.S., M.S., and Ph.D., in a row. Dr. Aljahdali has made research contributions on software testing, developing Software Reliability Models, Soft Computing for Software Engineering, Computer Security, Reverse Engineering and Medical Imaging. He is an author or co-author of over 40 peer reviewed 
academic publication. He is a member of professional societies like ACM, IEEE, Arab Computer Society (ACS) and International Society for Computers and Their Applications (ISCA). He is in the editorial board of many international journals like International Journal of Science and Advanced Technology, International Journal of Computer Technology and Applications, World of Computer Science and Information Technology, Journal of Intelligent Computing, International Journal of Computer Science \& Emerging Technologies, Universal Journal of Computer Science and Engineering Technology. He functioned as the General Chair, Program Chair, Session Chair, and member of the International Program Committee of the international conferences sponsored by various professional societies or organizations including the IEEE Computer Society, ACS and ISCA. He is also the conference chair of the forthcoming 24th International Conference on Computer Applications in Industry and Engineering (CAINE 2011) November 12-14, 2011, Honolulu, Hawaii, USA.

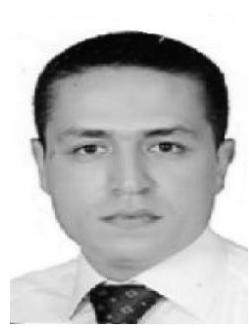

Omar Said is currently assistant professor at Dept. of Computer Science, Taif University, Taif, KSA. Also, he is an assistant professor in the Dept. of Computer Science, Menoufia University, Egypt. He received Ph.D degree from Menoufia University, Egypt. He has published many papers at international journals and conferences. His research areas are Computer Networking, Internet Protocols, Multimedia Communication, and wireless Communication, Internet of Things. 